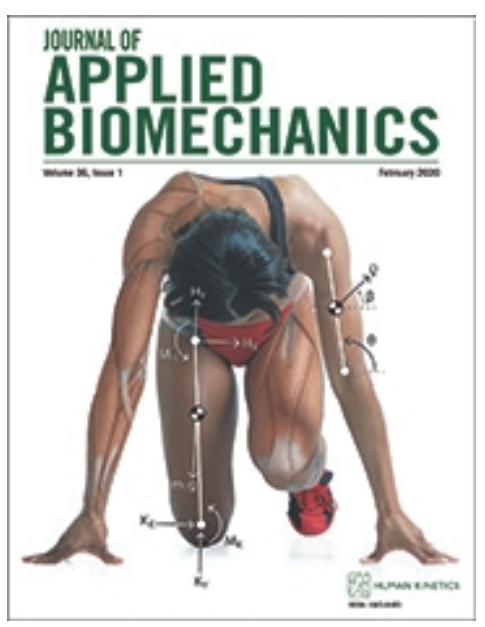

\title{
Comparison of transverse plane tibial and frontal plane rearfoot motion and movement coordination between runners with medial tibial stress syndrome and healthy controls
}

\begin{tabular}{|r|l|}
\hline Journal: & Journal of Applied Biomechanics \\
\hline Manuscript ID & JAB.2019-0367.R2 \\
\hline Manuscript Type: & Original Research \\
\hline Keywords: & running, kinematics, vector coding, statistical parametric mapping, injury \\
\hline
\end{tabular}

\section{SCHOLARONE \\ Manuscripts}


May 12, 2020

JAB.2019-0367.R2

Comparison of transverse plane tibial and frontal plane rearfoot motion and movement coordination between runners with medial tibial stress syndrome and healthy controls

Ben Langley $\mathrm{PhD}^{1}$, Nick Knight $\mathrm{MSc}^{2}$ and Stewart $\mathrm{C}$ Morrison $\mathrm{PhD}^{3}$

${ }^{1}$ Sport and Physical Activity, Edge Hill University, Ormskirk, Lancashire, UK

${ }^{2} \mathrm{NK}$ Active, Basingstoke, Hampshire, UK

${ }^{3}$ School Of Health Sciences, University of Brighton, Brighton, UK

Conflict of Interest Disclosure: None.

\section{Correspondence Address:}

Ben Langley $\mathrm{PhD}$

Department of Sport and Physical Activity

Edge Hill University

St Helens Road

Ormskirk, Lancashire ,UK, L39 4QP

Phone: 01695584880 Email: Ben.Langley@edgehill.ac.uk 


\section{Response to Editor and Reviewer Comments}

We would once again like to thank the editor and the reviewers for their constructive feedback on the revised manuscript. Please find out point by point responses to each comment below. Alterations within the manuscript are identified by underlined text.

\section{Editor Comments}

\begin{tabular}{|c|c|}
\hline Comment & Response \\
\hline $\begin{array}{l}\text { Please add a brief description of MTSS } \\
\text { (i.e. symptoms), perhaps as a sentence } \\
\text { starting on line } 42 \text {, for readers not } \\
\text { immediately familiar with it. }\end{array}$ & $\begin{array}{l}\text { We have added the following information } \\
\text { after the opening sentence in the } \\
\text { introduction to provide the suggested } \\
\text { information to readers. } \\
\text { "MTSS is associated with pain along the } \\
\text { posteromedial border of the distal aspect } \\
\text { of the tibia which occurs primarily during } \\
\text { running or exercise }{ }^{2 . "} \\
\text { Lines } 2-4\end{array}$ \\
\hline $\begin{array}{l}\text { Figure } 1 \text { appears to be highly similar to } \\
\text { a figure already published. If it is } \\
\text { modified, but similar, please indicate } \\
\text { this in the legend, citing the source } \\
\text { figure. If it is the same figure, } \\
\text { permission to reproduce this figure will } \\
\text { be required. } \\
\text { Weir, G., van Emmerik, R., Jewell, C., \& } \\
\text { Hamill, J. (2019). Coordination and } \\
\text { variability during anticipated and } \\
\text { unanticipated sidestepping. Gait \& } \\
\text { posture, } 67,1-8 .\end{array}$ & $\begin{array}{l}\text { We believe that the figure is unique } \\
\text { enough to not require permission to } \\
\text { reprint. We appreciate the figure is } \\
\text { similar to that in the Weir article, who's in } \\
\text { turn is similar to the one first presented } \\
\text { in Needham et al., } 2015 \text {. We have } \\
\text { however revised the figure legend to } \\
\text { better highlight the original source of the } \\
\text { information in the same manner as Weir. } \\
\text { We created the figure ourselves for this } \\
\text { publication and have superimposed our } \\
\text { own skeletal images on top to highlight }\end{array}$ \\
\hline
\end{tabular}




\begin{tabular}{|l|l|}
\hline & $\begin{array}{l}\text { the motion patterns associated with each } \\
\text { aspect of the plot. }\end{array}$ \\
\hline $\begin{array}{l}\text { The font sizes in Figures 2 and 3 are } \\
\text { quite small. Can the authors please } \\
\text { increase their size to improve } \\
\text { readability? }\end{array}$ & $\begin{array}{l}\text { We have further increased the font size } \\
\text { in the figures now, these are now set to } \\
\text { a minimal of size } 12 \text { font. Hopefully the } \\
\text { text will be more reader friendly now. }\end{array}$ \\
\hline
\end{tabular}




\section{Reviewer 1}

\begin{tabular}{|l|l|}
\hline \multicolumn{1}{|c|}{ Comment } & \multicolumn{1}{|c|}{ Response } \\
\hline $\begin{array}{l}\text { Thank you for the work updating the } \\
\text { analysis and manuscript. The revisions } \\
\text { are well done. }\end{array}$ & $\begin{array}{l}\text { We would like to take this opportunity to } \\
\text { once again thank the reviewer for their } \\
\text { constructive and insightful feedback on } \\
\text { the original manuscript. }\end{array}$ \\
\hline
\end{tabular}




\section{Reviewer 2}

\begin{tabular}{|c|c|}
\hline Comment & Response \\
\hline $\begin{array}{l}\text { I have a couple of minor comments } \\
\text { prior to publication. } \\
\text { There are a few instances where you } \\
\text { write "tibial" instead of "tibia" - eg. Lines } \\
208 \text { and } 214 \text {. }\end{array}$ & $\begin{array}{l}\text { We have checked the manuscript and } \\
\text { revised the terminology where } \\
\text { appropriate. This is highlighted by } \\
\text { underlined text within the manuscript, in } \\
\text { lines } 212,219 \text { and } 253 \text {. }\end{array}$ \\
\hline $\begin{array}{l}\text { Lines 113-114: You need to indicate to } \\
\text { the reader why you chose } 40 \mathrm{~Hz} \text {. So } \\
\text { state here that it was a hard setting in } \\
\text { the software. }\end{array}$ & $\begin{array}{l}\text { We have now added the following to the } \\
\text { end of the identified sentence; "using the } \\
\text { built-in hard setting within the Run3D } \\
\text { system" (Lines } 116-117 \text { ) }\end{array}$ \\
\hline $\begin{array}{l}\text { Please state how many trials you } \\
\text { averaged in your analysis (i.e. } 10 \text { stance } \\
\text { phases, } 20 \text { stance phases). Also make } \\
\text { it very clear that you didn't calculate the } \\
\text { coupling angle for each trial and then } \\
\text { average with circ stats - and that you } \\
\text { just measured the coupling angle on the } \\
\text { average CA for each subject and then } \\
\text { averaged that with circular stats. This } \\
\text { may have significantly impacted your } \\
\text { results. This should also be stated in } \\
\text { your limitations section where you } \\
\text { mention CV. }\end{array}$ & $\begin{array}{l}\text { Data were averaged over } 40-50 \text { stance } \\
\text { phases within the Run3D system, with } \\
\text { the variance linked to the system using a } \\
\text { time based metric to determine the } \\
\text { duration of the recording and differences } \\
\text { in step frequency between participants. } \\
\text { This information has now been added } \\
\text { within the method in line } 120 \text {. } \\
\text { We have made a minor modification to } \\
\text { the sentence describing how the } \\
\text { coupling angles were calculated to } \\
\text { further highlight that individual mean } \\
\text { motion patterns were used within the } \\
\text { calculation. The sentence on lines } 150 \text { - } \\
151 \text { now reads; } \\
\text { "The coupling angle was calculated } \\
\text { using equation } 1 \text { on an individual basis } \\
\text { using each participants' mean tibial } \\
\text { output by the Run3D system, with group }\end{array}$ \\
\hline
\end{tabular}




\begin{tabular}{|c|c|}
\hline & $\begin{array}{l}\text { mean coupling angles calculated using } \\
\text { circular statistics." } \\
\text { We have also highlighted that mean data } \\
\text { were extracted from the Run3D system } \\
\text { earlier within the method now to reiterate } \\
\text { this point (line 124). } \\
\text { We have added additional information } \\
\text { into the CV sentence within the } \\
\text { limitations section of the discussion to } \\
\text { identify the use of mean data to calculate } \\
\text { the coupling angle on a participant level } \\
\text { as limitation of the work. The sentence } \\
\text { now reads; } \\
\text { "Not being able to access the individual } \\
\text { trial data resulted in the need to utilise } \\
\text { participants mean motion patterns to } \\
\text { calculate the coupling angle and also } \\
\text { that movement coordination variability, } \\
\text { which has also been linked to injury } \\
\text { risk }{ }^{22} \text {, could not be calculated as this } \\
\text { requires the users to calculate the } \\
\text { coupling angle during each of the trials } \\
\text { recorded" (lines } 240 \text { - } 241 \text { ) }\end{array}$ \\
\hline $\begin{array}{l}\text { Lines 140-141: You calculated the T2 } \\
\text { statistic in this instance, so modify this } \\
\text { sentence to reflect that. }\end{array}$ & $\begin{array}{l}\text { Thank you for identifying this } \\
\text { terminological error. We have corrected } \\
\text { the sentence in line with your comment } \\
\text { (line 143). }\end{array}$ \\
\hline $\begin{array}{l}\text { Lines 152-155: This approach has } \\
\text { already been advanced in the Needham } \\
2015 \text { paper which is reference \#14. } \\
\text { Instead reference that paper. }\end{array}$ & $\begin{array}{l}\text { The highlighted aspect of the sentence } \\
\text { has been revised to cite the Needham } \\
\text { paper an reads; }\end{array}$ \\
\hline
\end{tabular}




\begin{tabular}{|c|c|}
\hline & $\begin{array}{l}\text { "Utilising the terminology proposed by } \\
\text { Needham et al }{ }^{14 " ~(L i n e ~ 155) ~}\end{array}$ \\
\hline $\begin{array}{l}\text { Figure } 3: \text { The * on the graph is missing } \\
\text { to indicate the significant difference } \\
\text { between in-phase tibia. }\end{array}$ & $\begin{array}{l}\text { Thank you for the diligent review and } \\
\text { picking up on this. We have now added } \\
\text { the * to figure } 3 \text {. }\end{array}$ \\
\hline $\begin{array}{l}\text { Discussion } \\
\text { Lines } 217-220 \text { : I apologize I didn't pick } \\
\text { up on } \\
\text { this in the last review - I wonder } \\
\text { whether this statement is a little bold as } \\
\text { there is an abundance of evidence } \\
\text { stating the opposite. Soften these } \\
\text { conclusions and incorporate other } \\
\text { evidence to present both perspectives. }\end{array}$ & $\begin{array}{l}\text { We appreciate that the statement does } \\
\text { go against the typically approaches } \\
\text { utilised which largely, in our opinion, are } \\
\text { developed off the back of traditional } \\
\text { running injury paradigms and that the } \\
\text { wording of the identified section may be } \\
\text { excessively bold. As such we have } \\
\text { softened this section and clearly } \\
\text { highlighted that this suggestion } \\
\text { contrasting what would typically be } \\
\text { promoted. } \\
\text { "In contrast to conventional approaches } \\
\text { which would look to reduce rearfoot } \\
\text { eversion in line with tradition running } \\
\text { injury paradigms, these findings suggest } \\
\text { interventions which look to increase the } \\
\text { duration of rearfoot eversion may } \\
\text { actually be beneficial for the MTSS } \\
\text { group by improving the in-phase } \\
\text { coordination of the rearfoot and tibia, in } \\
\text { turn reducing the torsional stress placed } \\
\text { upon the tibia which may decrease the } \\
\text { likelihood of developing MTSS. This } \\
\text { suggestion, while based on the evidence } \\
\text { gathered within this study, is however } \\
\text { speculative and requires further } \\
\text { exploration." Lines } 220-221 \& 225 \text { - } 226\end{array}$ \\
\hline
\end{tabular}


May 13, 2020

2

JAB.2019-0367.R2

3

4 Comparison of transverse plane tibial and frontal plane rearfoot motion and movement

5 coordination between runners with medial tibial stress syndrome and healthy controls

6

7 Ben Langley $\mathrm{PhD}^{1}$, Nick Knight $\mathrm{MSc}^{2}$ and Stewart $\mathrm{C}$ Morrison $\mathrm{PhD}^{3}$

$8{ }^{1}$ Sport and Physical Activity, Edge Hill University, Ormskirk, Lancashire, UK

$9 \quad{ }^{2} \mathrm{NK}$ Active, Basingstoke, Hampshire, UK

$10{ }^{3}$ School Of Health Sciences, University of Brighton, Brighton, UK

11

12 Conflict of Interest Disclosure: None.

13

14 Correspondence Address:

15 Ben Langley $\mathrm{PhD}$

16 Department of Sport and Physical Activity

17 Edge Hill University

18 St Helens Road

19 Ormskirk, Lancashire, UK, L39 4QP

20 Phone: 01695584880 Email: Ben.Langley@edgehill.ac.uk 


\section{Abstract}

22 Medial tibial stress syndrome (MTSS) is a common running related injury. Alterations in 23 movement patterns and movement coordination patterns have been linked to the development 24 of overuse injuries. The aim of this study was to compare transverse plane tibial and frontal plane rearfoot motion and the coordination of these movements between runners with MTSS and healthy controls. Ten recreational runners with MTSS and ten healthy controls ran at $11 \mathrm{~km} . \mathrm{hr}^{-1}$ on a treadmill. A three-camera motion analysis system, operating at $200 \mathrm{~Hz}$, was used to calculate tibia and rearfoot motion. Stance phase motion patterns were compared between groups using multivariate analysis; specifically, Hotelling's $\mathrm{T}^{2}$ test with statistical parametric mapping (SPM1D). A modified vector coding technique was used to classify the coordination of transverse plane tibial and frontal plane rearfoot motion. The frequency of each coordination pattern displayed by each group was compared using independent samples t tests. Individuals with MTSS displayed significantly $(p=.037, \mathrm{~d}=1.00)$ more anti-phase coordination (tibial internal rotation with rearfoot inversion) despite no significant $(p>.05)$ differences in stance phase kinematics. The increased anti-phase movement may increase the torsional stress placed upon the medial aspect of the tibia contributing to the development of MTSS.

Keywords: running; injury; vector coding; statistical parametric mapping; kinematics 
Medial tibial stress syndrome (MTSS) is one of the most common lower limb injuries associated with recreational running, with incidence rates between $13.6 \%$ and $20 \%{ }^{1}$. MTSS is associated with pain along the posteromedial border of the distal aspect of the tibia which occurs primarily during running or exercise ${ }^{2}$. Whilst the aetiology of MTSS remains difficult to determine ${ }^{2,3}$, there have been considerable efforts to identify the risk factors associated with developing this injury. Systematic reviews ${ }^{3-5}$ have been published which identify increased body mass index, plantarflexion range of motion, hip internal/external rotation range of motion, reduced lean calf girth and a pronated foot type as intrinsic risk factors associated with the development of $\mathrm{MTSS}^{3-5}$. The majority of existing studies have explored the relationship between static measures and MTSS, the relationship between dynamic foot motion and MTSS has received less attention within the literature.

Traditional running injury paradigms link excessive rearfoot pronation with the development of running related injuries ${ }^{7,8}$, on the premise that increasing the magnitude or duration of pronation would lead to abnormal loading or stresses being applied to the lower limb, in particular the tibia ${ }^{9}$. Increased rearfoot eversion or eversion velocity, measures typically used to quantify the magnitude and rate of foot pronation, have been reported in participants who had or later developed lower limb injuries compared to healthy controls during running ${ }^{8-10}$. Comparable findings have been reported within studies which compared RF eversion between healthy controls and MTSS groups during walking ${ }^{11}$ and running ${ }^{6}$. While this information provides some evidence linking rearfoot eversion and MTSS specifically, these studies have compared discrete variables extracted from stance phase kinematics between healthy and MTSS or injured populations. More advanced statistical methods, such as statistical parametric mapping (SPM), enable the comparison of kinematic waveforms, in turn 
providing a more in-depth comparison of movement patterns between populations and removing the need to subjectively preselect variables of interest ${ }^{12}$.

Furthermore, no studies, to the authors knowledge, have explored tibial rotations between healthy and MTSS groups. Rearfoot eversion has been shown to be associated with tibial internal rotation due to the coupling of the calcaneus and the tibia, via the talus ${ }^{7}$. As such increased rearfoot eversion may be accompanied by increased internal rotation of the tibia. Concurrent exploration of both rearfoot and tibial kinematics would enable the coupling between these motions to be compared between MTSS and healthy cohorts. Exploration of joint coupling has previously been proposed and utilised as a means of evaluating injury aetiology ${ }^{13}$ and may help to elucidate potential mechanisms for the development of MTSS. Relatively recent advancements in vector coding techniques ${ }^{14-16}$ enable the coordination of joint couples to be explored from angle-angle plots, with coordination patterns being described as either in-phase (two segments rotating in the same direction) or anti-phase (two-segments rotating in opposite directions), with proximal or distal segment dominance (Figure 1).

Therefore, the aim of this paper was to compare transverse plane tibial and frontal plane rearfoot motion and the coordination of these movements between runners with MTSS and healthy controls. Three hypotheses were tested within this study; tibial internal rotation and rearfoot eversion would be greater in the MTSS group, and the coordination of transverse plane tibial and frontal plane rearfoot motions would differ between MTSS and healthy groups.

\section{Methods}

A total of 20 male recreational runners participated in this study, comprised of 10 runners (age: $32 \pm 8 \mathrm{y}$; height: $1.80 \pm 0.05 \mathrm{~m}$; mass; $78 \pm 8 \mathrm{~kg}$ ) with MTSS and 10 pain free controls (age: $34 \pm 9 \mathrm{y}$; height: $1.76 \pm 0.10 \mathrm{~m}$; mass; $75 \pm 10 \mathrm{~kg}$ ). Inclusion in the study required 
all participants to be; male, aged between $18-45$ years, running a minimum of $15 \mathrm{k}$ per week, comfortable running on a treadmill, pain when running $2 / 10$ or below on a visual analogue scale, not wearing orthoses, no history of gait retraining, or other musculoskeletal injury other than MTSS at the time of testing. Inclusion in the MTSS group were in line with the inclusion criteria and pre-existing criteria described by Winters et a ${ }^{17}$ for MTSS. Specifically, the MTSS group displayed pain along the lower medial boarder of the tibia for more than $5 \mathrm{~cm}$ upon palpation which was assessed prior to commencement of testing sessions for this group by a trained podiatrist $(\mathrm{NK})$ at the commencement of testing for those within this group. Prior to data collection participants provided written informed consent form and ethical approval for the study was granted by the University of Brighton School of Health Sciences Research Committee.

Participants attended a single testing session lasting approximately 45 minutes in which they were asked to run in their own running shoes, at a standardised speed of $11 \mathrm{~km} \cdot \mathrm{hr}^{-1}$, to remove any potential speed related changes in kinematic profiles, for 5 minutes on a Sole F65 treadmill (Sole Fitness, Salt Lake City, Utah, USA). Kinematic data were collected continuously for the first 30 seconds of the final minute of the run. Data collection was undertaken at this time point to provide participants with a familiarisation period to the treadmill in order to reduce discrepancies between treadmill and overground kinematics ${ }^{18}$.

Kinematic data were collected using a Run3D automated motion capture system (Run3D Oxford, UK) which consists of three VICON Bonita cameras (Oxford, United Kingdom), sampling at 200Hz. The Run3D system was created to enable 3D motion capture within clinical settings, as such data processing and kinematic modelling are largely automated. Initially, the Run3D system reconstructed and labelled marker trajectories over the entire recording, in this case 30 seconds. Gaps within the marker trajectories were filled using cubic Bezier patches and any trials with excessive marker loss, $50 \%$ or above, were deemed invalid 
and data was recaptured. Prior to data collection the position of the VICON cameras were optimised to minimise marker loss and tracking markers attached to the tibia and rearfoot were visible throughout the stance phase during pilot assessments. Once marker trajectories were labelled and gap filled, they were low pass filtered at $40 \mathrm{~Hz}$ using the built-in hard setting within the Run3D system. Trials were then partitioned into gait cycles based on the vertical position and orientation of the rearfoot segment, with gait cycles exceeding $\pm 10 \%$ of the median gait cycle length removed. Euler angles were calculated utilising an XYZ cardan sequence of rotations, before mean joint rotations across all remaining gait cycles $(40-50$ gait cycles perparticipant) were calculated. Any gait cycles in which the root mean square value was greater than three standard deviations from the mean were removed at this point by the software, before the mean and standard deviation values were updated and output. Within this study we extracted mean frontal plane rearfoot and transverse plane tibial kinematics for each participant analysis, removing swing phase data and time normalising the remaining output to 101 data points corresponding to $100 \%$ stance phase duration. All data presented is for the right limb as this was the limb classified as displaying symptoms of MTSS for this group. attached bilaterally to the lower limbs, in line with the model described by Ferber et al ${ }^{19}$. Of specific interest to this study, the tibia was defined proximally using markers located on the medial and lateral femoral epicondyles, and distally by markers located on the medial and lateral malleoli. The tibia was tracked using cluster of four non-colinear markers attached to a rigid plastic shell and attached to the posterior-lateral aspect of the segment. The rearfoot was collection the Run 3D system was calibrated in line with the manufactures guidelines, only defined by two markers placed on vertically on the central aspect of the shoes heel counter, and a third marker located on the lateral aspect of the rear aspect of the shoe. Prior to data collection the Run 3D system was calibrated in line with the manufactures guidelines, only calibrations resulting in residuals of $<0.1$ were accepted. A static trial was recorded with 
participants standing in a relaxed bipedal stance, with the longitudinal axis of each foot $26 \mathrm{~cm}$ apart and the feet in a neutral alignment; this orientation was standardised using a calibration mat.

Multivariate statistical analysis was undertaken using two-sample Hotelling's $\mathrm{T}^{2}$ test with SPM to compare transverse plane tibial and frontal plane rearfoot motion between the MTSS and control groups. SPM calculates a statistical parametric map by plotting the $\underline{T}^{2}-$ statistic for each time point within the data set and applies random field theory to determine if the average gradient of the t-statistic and clusters of points are above the critical threshold, in turn identifying $\mathrm{p}$ values below $0.05^{12}$. SPM analysis was undertaken in Python using publicly available scripts developed by Pataky ${ }^{20}$.

The modified vector coding technique described by Needham et al ${ }^{14,15}$ was used to quantify the coordination between transverse plane tibial and frontal plane rearfoot motion. The coupling angle was calculated using equation 1 on an individual basis using each participants' mean tibial rotation and rearfoot motion patterns output by the Run3D system, with group mean coupling angles calculated using circular statistics.

Eq. 1 Coupling angle $=\operatorname{atan}\left(\frac{\theta_{\text {rearfoot }(i+1)}-\theta_{\text {rearfooti }}}{\theta_{\text {tibia }(i+1)}-\theta_{\text {tibiai }}}\right)$

Coupling angles were corrected to provide values between $0^{\circ}$ and $360^{\circ}$ according to Needham et $\mathrm{al}^{15}$. Utilising the terminology proposed by Needham et $\mathrm{al}^{14}$, coordination patterns were classified into one of eight categories based on whether the movements of the tibia and rearfoot were in-phase or anti-phase, with proximal or distal dominance and the direction of the rotations (Figure 1). The percentage of stance spent in each of these categories was calculated and compared statistically using independent samples t tests using SPSS Version 23 (IBM, Chicago, Il). No correction for multiple comparisons were made to the alpha level, 
which was set at $p<0.05$. Cohen's $d$ was also calculated to provide an estimate of effect sizes and interpreted as follows; small (0.2), moderate (0.5) and large (0.8) effect ${ }^{21}$.

\section{Results}

No significant $(p>.050)$ differences in transverse plane tibial or frontal plane rearfoot motion were reported between the control and MTSS groups during the stance phase of running gait (Figure 2). A significant difference $(p=.037, \mathrm{~d}=1.00)$ in transverse plane tibial and frontal plane rearfoot coordination pattern was reported, with the frequency of anti-phase coordination with tibial dominancy (tibial internal rotation with rearfoot inversion) was greater in the MTSS

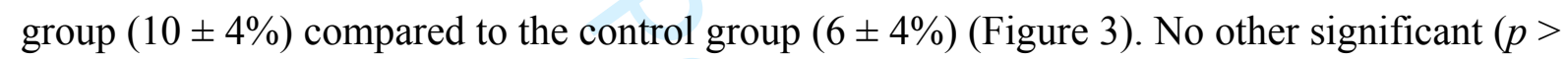
$.050, \mathrm{~d}=0.0-0.4)$ differences in the frequency of the remaining coordination pattern classifications were reported between the control and MTSS groups.

\section{Discussion}

The aim of this study was to compare transverse plane tibial and frontal plane rearfoot motion and the coordination of these movements between runners with MTSS and healthy controls. The findings reject the first two hypotheses proposed with no statistically significant differences in stance phase transverse plane tibia or frontal plane rearfoot motion between the MTSS and control groups. In contrast, the final hypothesis was supported with those in the MTSS group displaying significantly more anti-phase movement, with tibial dominancy, compared to the control group. The findings of this study therefore demonstrate that the coordination, or coupling, pattern of the tibia and rearfoot differ between those with MTSS and 
healthy controls; even in the absence of any significant changes in tibial or rearfoot motion patterns.

The rearfoot eversion motion patterns displayed by the MTSS and healthy control groups within this study (Figure 2B) were comparable and these contradict the findings of previous studies, ${ }^{6,8,9}$, which reported significantly increased rearfoot eversion in injured populations compared to healthy controls during running. The disparity between our findings and the previous literature ${ }^{6,8,9}$ is likely due to the inclusion criteria for the injured group. While the present study compared those with MTSS alone to a healthy control group, all the previous studies ${ }^{6,8,9}$ have utilised injured populations which do not exclusively contain individuals with MTSS. Pooling of multiple injuries into a single injured population is likely to introduce a cross over effect with risk factors associated with one condition masking those associated with another. As such it seems pertinent, especially due to the discrepancies in the findings identified, that comparisons are made between populations with a specific injury and healthy controls as this may help to better understand the aetiological risk factors associated with that injury, which may in turn help to develop more specific and successful (p)rehabilitation interventions.

Despite a lack of significant changes in tibial internal rotation or rearfoot eversion between groups, the MTSS did display significantly altered coordination patterns. This finding suggests that it is the coordination of the movement between the tibia and rearfoot, as opposed to the discrete motion of either of these segments, which is potentially more important to understand the development of MTSS. Increased anti-phase movement in the MTSS group, which appears to be due to increases in the anti-phase movement around 30$40 \%$ of the stance phase (based on visual assessment of Figure 3), may increase torsional stresses placed upon the tibia at this time point, as the rearfoot begins to invert while the tibia continues to internally rotate. Interestingly, this finding conflicts with traditional injury 
paradigms which link excessive eversion and tibial internal rotation to the development of running injuries. Excessive eversion has been assumed to result in increased tibial internal rotation which would result in an in-phase movement coordination pattern rather than the anti-phase pattern displayed by the MTSS group within this study. However, logically increasing the torsional stress placed upon the tibia would likely lead to higher forces acting upon the medial aspect of the bone and in turn increasing the risk of MTSS. Further work is required to explore whether the increased anti-phase movement does relate to significant increases in torsional stresses.

While traditional approaches to reducing running injury risk have focused on reducing rearfoot eversion through footwear or orthotic interventions ${ }^{7}$, this may potentially increase the anti-phase coordination pattern displayed by the MTSS group. Visual inspection of Figure 2B shows that the MTSS group begin to invert before $40 \%$ of the stance phase, yet the tibia continues internally rotating until closer to $50 \%$ of the stance phase. The impact of the prolonged tibial internal rotation upon the joint coupling is evident within the angle-angle plot displayed in Figure 2C. In contrast to conventional approaches which would look to reduce rearfoot eversion in line with tradition running injury paradigms, these findings suggest interventions which look to increase the duration of rearfoot eversion may actually be beneficial for the MTSS group by improving the in-phase coordination of the rearfoot and tibia, in turn reducing the torsional stress placed upon the tibia which may decrease the likelihood of developing MTSS. This suggestion, while based on the evidence gathered within this study, is however speculative and requires further exploration MTSS group were recruited on the basis they had this condition at the time of testing and as such there is the possibility that the movement and coordination patterns displayed by this group are a result of, as opposed to the cause of, this injury. However, a movement strategy 
which increases the anti-phase movements of the tibia and rearfoot seems an unlikely preventative solution once MTSS has been developed, as the opposing movement of the two segments around midstance would likely increase the torsional stresses placed upon the tibia and in turn increase the risk of injury. Larger scale case control and more prospective studies designs would be required to confirm this hypothesis. The use of the Run3D system is another potential limitation of this study. As detailed within the method section, the Run3D system is designed to be used within a clinical setting, to decrease the time constraints placed upon users, and as such the system outputs mean and standard deviation values only for the trials recorded, while also limiting the user's ability to manipulate the data processing pipeline. Not being able to access the individual trial data resulted in the need to utilise participants mean motion patterns to calculate the coupling angle and also that movement coordination variability, which has also been linked to injury risk ${ }^{22}$, could not be calculated as this requires the users to calculate the coupling angle during each of the trials recorded. Additionally, footwear was not standardised within this study and variance in the stability features built into different participants running shoes may have influenced kinematic patterns in different ways.

The findings of this study suggest that tibial internal rotation and rearfoot eversion do not differ significantly between individuals with MTSS and healthy controls. However, the MTSS group displayed significantly increased anti-phase coordination with tibial dominancy during the stance phase of the running gait cycle. The significant increase in anti-phase motion displayed by the MTSS groups appears to be related to the rearfoot beginning to invert while the tibia is still internally rotating, which would likely increase the torsional stress placed upon the tibia. Interventions which improve the coupling of rearfoot eversion and tibial internal rotation may help to reduce the risk of developing MTSS, by increasing the in-phase movements of these segments. 


\section{Acknowledgements}

259

260

261

262

263

264

265

266

267

268

269

270

271

272

273

We would like to thank Run3D for providing additional information relating to the processing of data within their software, which helped to increase the clarity of the manuscript.

\section{References}

1. Lopes $A D$, Hespanhol Jr LC, Yeung $S C$ et al. What are the main running-related musculoskeletal injuries? A systematic review. Sports Med 2012; 42(10):891-905.

2. Moen MD, Tol JL, Steunebrink $M$ et al. Medial tibial stress syndrome: a critical review. Sports Med 2009; 39(7):523-546.

3. Hamstra-Wright KL, Bliven KC, Bay C. Risk factors for medial tibial stress syndrome in physically active individuals such as runners and military personnel: a systematic review and meta-analysis. Br J Sports Med 2015; 49(6):362-369.

4. Brune SG, Khan KM, Boudville PB et al. Risk factors associated with exertional medial tibial pain: a 12 month prospective clinical study. Br J Sports Med 2004; 38(4):441445.

5. Newman $\mathrm{P}$, Witchalls $\mathrm{J}$, Waddington $\mathrm{G}$ et al. Risk factors associated with medial tibial stress syndrome in runners: a systematic review and meta-analysis. Open Access $J$ Sports Med 2013; 13(4):229-241.

6. Becker J, James S, Warner R et al., Biomechanical factors associated with achilles tendinopathy and medial tibial stress syndrome in runners. Am J Sports Med 2017; 45(11): 2614-2621.

7. Stacoff A, Nigg BM, Reinschmidt $C$ et al., Tibiocalcaneal kinematics of barefoot versus shod running. J Biomech 2000; 33(11):1387-1395.

8. Bramah C, Preece SJ, Gill N et al. Is there a pathological gait associated with common soft tissue running injuries? AM J Sports Med 2018; 46(12):3023-3031. 
9. Willems TM, De Clercq D, Delbaere $\mathrm{K}$ et al. A prospective study of gait related risk factors for exercise-related lower leg pain. Gait Posture 2006; 23(1):91-98.

10. Kuhman DJ, Paquette MR, Peel SA et al. Comparison of ankle kinematics and ground reaction forces between prospectively injured and uninjured collegiate cross country runners. Hum Move Sci 2016; 47:9-15.

11. Akiyama K, Noh B, Fukano M et al. Analysis of the talocrural and subtalar joint motions in patients with medial tibial stress syndrome. J Foot Ankle Res 2015; 8: 25.

12. Pataky TC, Robinson MA, Vanrenterghem J. Vector field statistical analysis of kinematic and force trajectories. J Biomech 2013; 46(14):2394-2401.

13. Rodrigues $P$, Chang $R$, TenBroek $T$ et al. Evaluating the coupling between foot pronation and tibial internal rotation continuously using vector coding. J Appl Biomec 2015; 31(2):88-94.

14. Needham RA, Naemi R, Chockalingam N. A new coordination pattern classification to assess gait kinematics when utilising a modified vector coding technique. J Biomech 2015; 48(12):3506-3511.

15. Needham RA, Naemi R, Chockalingam N. Quantifying lumbar-pelvis coordination during gait using a modified vector coding technique. J Biomech 2014; 47(5):10201026.

16. Chang R, Van Emmerik R, Hamill J. Quantifying rearfoot-forefoot coordination in human walking. J Biomech 2008; 41(14):3101-3105.

17. Winters M, Bakker EWP, Moen MH et al. Medial tibial stress syndrome can be diagnosed reliably using history and physical examination. Br J Sports Med 2018; 52(19):1267-1272.

18. Riley PO, Dicharry J, Franz J et al. A kinematics and kinetic comparison of overground and treadmill running. Med Sci Sports Exerc 2008;40(6):1093-1100.

19. Ferber R, McClay Davis I, Williams DS et al. A comparison of within- and between-day reliability of discrete 3D lower extremity variables in runners. J Orthop Res 2002; 20(6):1139-1145. 
20. Pataky T. 2019. SPM1D One- and two-sample tests. Available at http://www.spm1d.org/doc/Stats1D/onetwosample.html. Accessed 15 August 2019.

21. Cohen J. Chapter 1 - The Concepts of Power Analysis, in Statistical Power Analysis for the Behavioral Sciences, $2^{\text {nd }}$ ed., New York, Routledge, 2013.

22. Hamill J, Palmer C, Van Emmerik RE. Coordinative variability and overuse injury. Sports Med Arthrosc Rehabil Ther Technol 2012; 4(1):45. 
322 Figure 1. Coordination pattern classification based on the coupling angle displayed as a polar 323 plot using the terminology described by Needham et al., ${ }^{14}$. Visual illustrations of the segment 324 motions associated with each quadrant of the polar plot are overlaid. NOTE: At $0^{\circ}$ and $180^{\circ}$ 325 the proximal segment is rotating with no movement of the distal segment, and at $90^{\circ}$ and $270^{\circ}$ the distal segment is rotating with no movement of the proximal segment

Figure 2. (A) Transverse plane tibial and (B) frontal plane rearfoot motion, (C) angle-angle diagram during the stance phase of running gait for the control (black line) and medial tibial stress syndrome (MTSS) (grey line) groups. (D) $\mathrm{T}^{2}$ statistic from SPM analysis and critical threshold (horizontal dashed lines) displayed.

333 Figure 3. Mean coupling angle during the stance phase of running gait for the control (black) 334 and medial tibial stress syndrome (MTSS) (grey) groups, and the frequency with which each coordination pattern is evident throughout the stance phase. ${ }^{*} p<0.05$ 


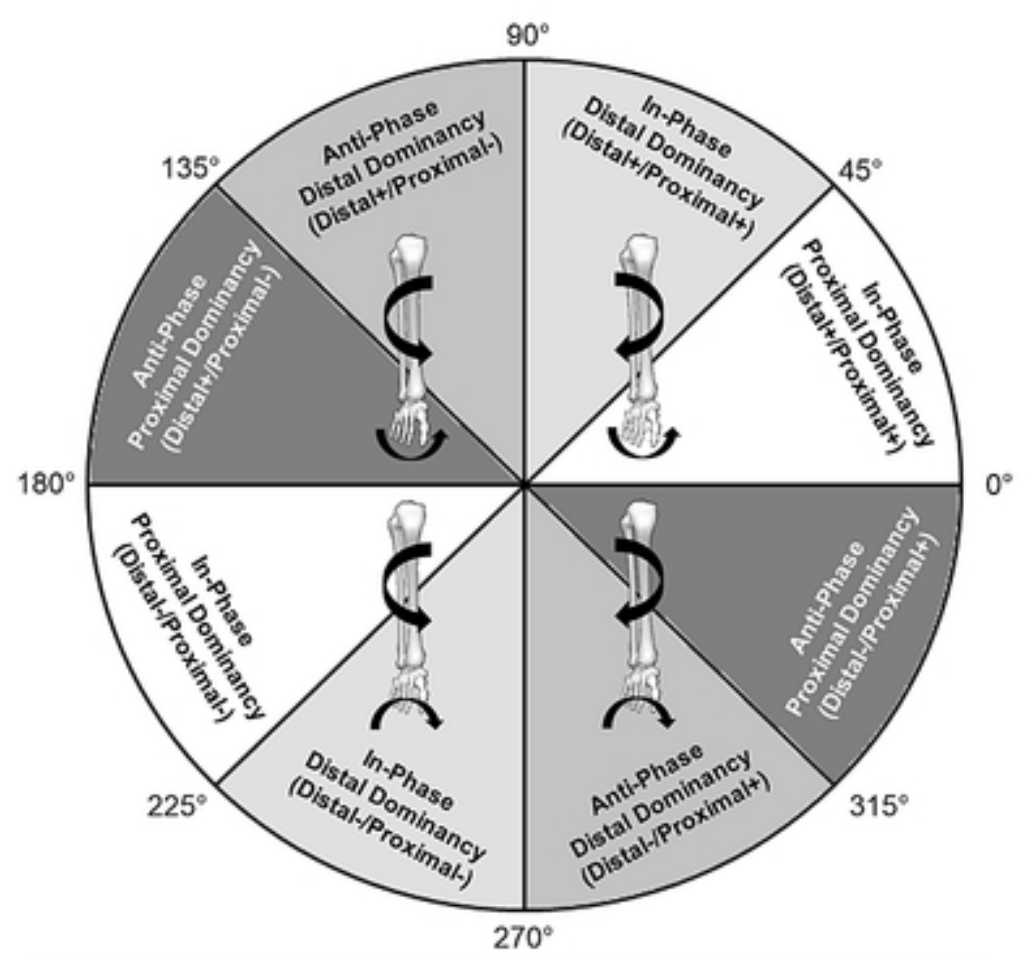

Figure 1. Coordination pattern classification based on the coupling angle displayed as a polar plot using the terminology described by Needham et al.,14. Visual illustrations of the segment motions associated with each quadrant of the polar plot are overlaid. NOTE: At $0^{\circ}$ and $180^{\circ}$ the proximal segment is rotating with no movement of the distal segment, and at $90^{\circ}$ and $270^{\circ}$ the distal segment is rotating with no movement of the proximal segment

$34 \times 31 \mathrm{~mm}(300 \times 300 \mathrm{DPI})$ 

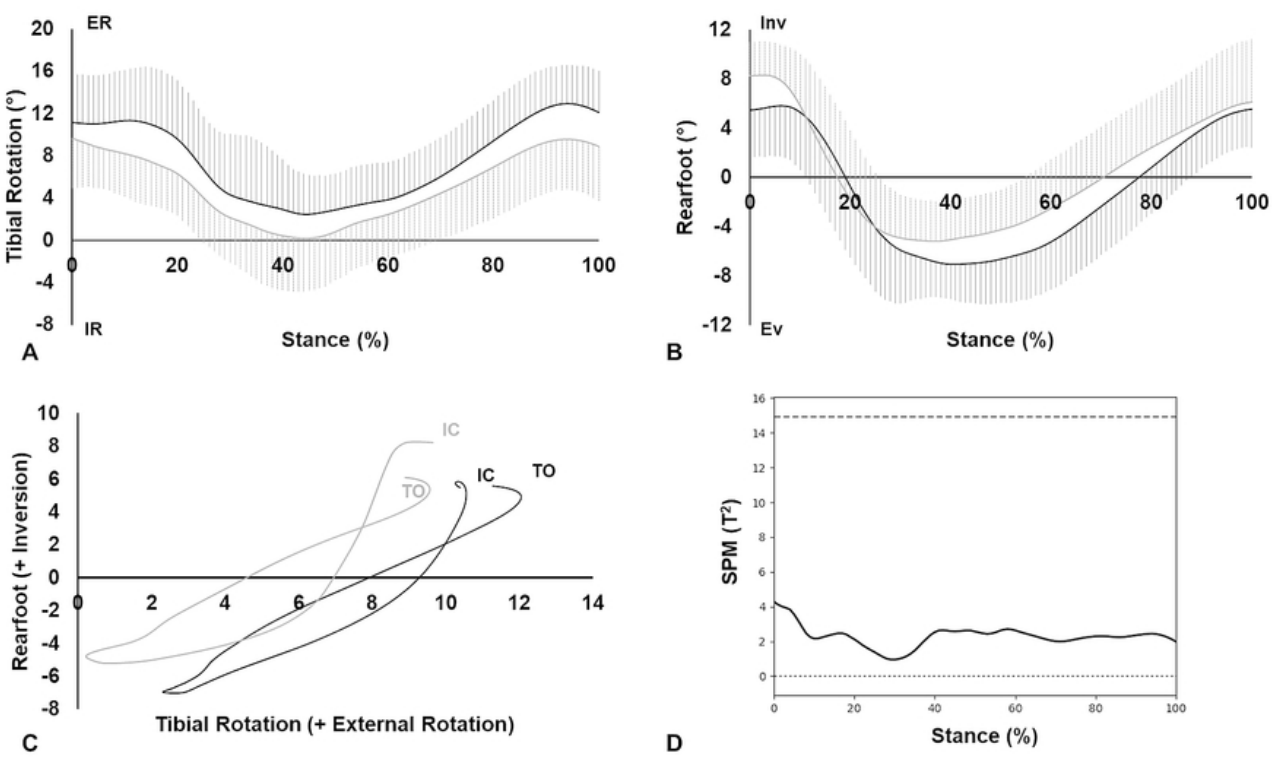

Figure 2. (A) Transverse plane tibial and (B) frontal plane rearfoot motion, (C) angle-angle diagram during the stance phase of running gait for the control (black line) and medial tibial stress syndrome (MTSS) (grey line) groups. (D) T2 statistic from SPM analysis and critical threshold (horizontal dashed lines) displayed.

$75 \times 44 \mathrm{~mm}(300 \times 300$ DPI $)$ 

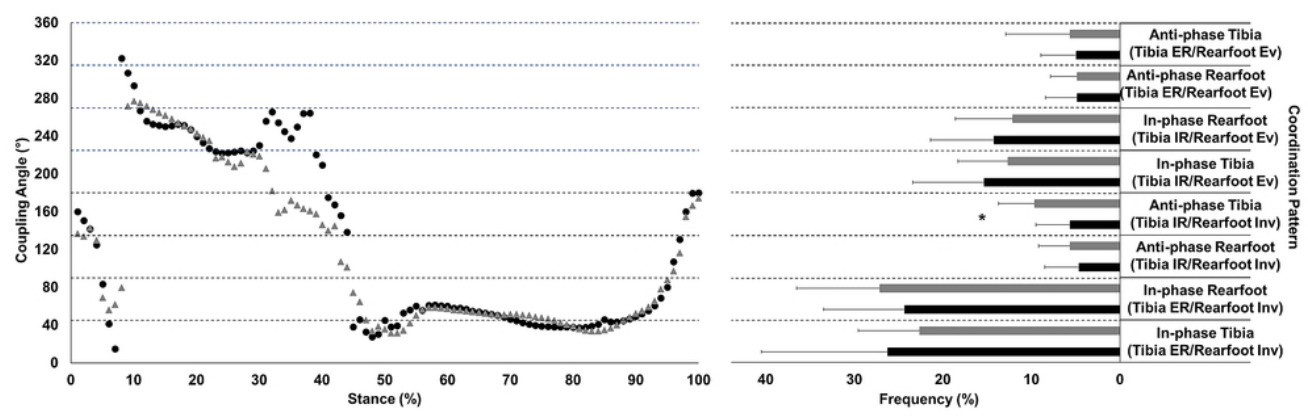

Figure 3. Mean coupling angle during the stance phase of running gait for the control (black) and medial tibial stress syndrome (MTSS) (grey) groups, and the frequency with which each coordination pattern is evident throughout the stance phase. $* p<0.05$

$83 \times 27 \mathrm{~mm}(300 \times 300 \mathrm{DPI})$ 